\section{SAS procedures for approximate randomization tests}

\author{
RU SAN CHEN and WILLIAM P. DUNLAP \\ Tulane University, New Orleans, Louisiana
}

Approximate randomization tests are alternatives to conventional parametric statistical methods used when the normality and homoscedasticity assumptions are violated. This article presents an SAS program that tests the equality of two means using an approximate randomization test. This program can serve as a template for testing other hypotheses, which is illustrated by modifications to test the significance of a correlation coefficient or the equality of more than two means.

Conventional parametric statistical methods typically have been applied for hypothesis testing in behavioral research. These tests require two basic assumptions: the observations are drawn from normally distributed populations, and the population variances are equal. When these assumptions are violated, several choices are available. First, a researcher may rely on the robustness of the parametric tests, trusting that assumption violations should have little effect on the power or Type I error rates for the tests. A number of studies, however, have challenged the robustness of the parametric tests under assumption violations. For instance, distorted Type I error rates for analyses of variance on data drawn from mixed normal distribution were reported by Bradley (1968, 1978), and loss of power for the $t$ test on skewed data with unequal group sizes was shown by Toothaker (1972).

The second choice is to transform the data to reduce skewness or the discrepancy in variances. Two drawbacks are associated with this approach: it is often difficult to select an appropriate transformation (see, e.g., Dixon \& Massey, 1969), and if the transformation has been found, the hypotheses tested before and after transformation may be different (Games, 1984).

The third choice is to employ nonparametric methods based on the rank transformation, such as the WilcoxonMann-Whitney test for two independent samples or the Kruskal-Wallis One-Way Analysis of Variance by Ranks test (Siegel \& Castellan, 1988). Although these tests have been shown to be powerful, loss of information by replacing the raw data by their ranks may lead to loss of power in certain circumstances. Another problem with this approach is that too many ties in the ranks may lower their power (Manly, 1991, p. 52).

This paper benefited from many helpful comments and suggestions from two anonymous reviewers. Correspondence should be addressed to Ru San Chen, Department of Psychology, Tulane University, New Orleans, LA 70118.
Randomization tests have been recommended as alternatives to parametric tests when the standard assumptions are violated (e.g., Edgington, 1987; Manly, 1991; Noreen, 1989). The idea of randomization tests, first described by Fisher (1935), is relatively simple. Suppose we want to test the null hypothesis of no mean difference between two groups. If the subjects of the two groups are actually from the same population, any rearrangement of the group membership should have little effect on mean difference. Let $M_{0}$ be the mean difference for the observed data. $M_{0}$ should not be very extreme in the distribution of $M$, mean difference for all possible permutations of the observed data. The significance level of $M_{0}$ is the percentage of the values in $M$ that are equal to or more extreme than $M_{0}$. Randomization tests evaluate significance on the basis of the observed data; therefore, assumptions about populations are not required. The randomization test, which is distribution free and preserves original data values, is a serious competitor to alternative tests.

One drawback of the randomization tests described above, sometimes called exact permutation tests, is that as the sample sizes grow, the number of possible permutations increases rapidly. Even with modern computers, exact tests are often not feasible. To make the tests feasible, the approximate randomization test (ART) has been proposed (e.g., Edgington, 1987; Noreen, 1989). The ART is a test that randomly draws a subset from all possible permutations to estimate the exact significance level of the statistic. Edgington (1969) analytically proved that the ART with 999 random samples with replacement was as powerful as the exact permutation test. Manly (1991, p. 37) and Noreen $(1989$, p. 15$)$ also recommend that 1,000 random samples would be appropriate to obtain a significance level similar to that obtained by the exact permutation test.

The power of randomization tests compared with their counterpart parametric and nonparametric methods have been evaluated in a number of studies. Hoeffding (1952) found that the powers of randomization tests were asymptotically similar to related conventional parametric tests when the standard assumptions were met. Noreen (1989, p. 35) compared the power of the ART and the parametric $t$ test for a two-sample location problem and for the significance of a Pearson correlation coefficient, and concluded that the two tests had similar power for normally distributed data.

Still and White (1981) and Bradbury (1987) reported power advantages for the ART over the parametric $F$ test for data from mixed normal distributions. Toothaker (1972) concluded that for skewed data, randomization tests had higher power than Student's $t$ test if the means were positively related to the sample sizes. Edgington (1987, p. 94) also reported that randomization tests were more powerful than the parametric $t$ test with certain skewed 
data. Kempthone and Doerfler (1969) found superior power for randomization tests over the Wilcoxon Signed Ranks Test under eight distributions. Because the powers of ARTs are different for various tests and the assumptions can be violated in different ways, further research is needed before a more general statement can be made about the power comparisons between ARTs and their counterpart methods.

In summary, the ART procedures can be considered for hypothesis testing whenever the normality and/or the homoscedasticity assumptions appear to be violated. Micceri (1989) recently investigated 440 large-sample achievement and psychometric measures and found that all of them had nonnormal distributions. Therefore, ARTs should be among the data-analysis tools available to behavioral researchers. There is no reason for applying a randomization test if the assumptions of a conventional test are met, although a randomization test will yield similar results under such circumstances.

The general steps for the ART are as follows:

1. Select an appropriate statistic and compute the statistic for the original sample.

2. Shuffle the raw data (i.e., random permutation) and form a pseudosample with the same size as the original one. Repeat this procedure to obtain a desired number of pseudosamples (NOP).

3. Compute the pseudostatistic for each pseudosample.

4. Count the number of pseudostatistics that are equal to or more extreme than the original statistic (number of significant cases, or NOS). The ratio NOS/NOP is the estimated significance level, the $p$ value, of the test.

Ordinarily, the estimated significance level, the $p$ value, is used directly to make the judgment regarding significance: if $p$ is less than $\alpha$, the result is significant; if $p$ is greater than $\alpha$, it is not significant.

A further refinement, however, was proposed by Noreen (1989, p 56). Let $\phi$ be the actual significance level, and then $\phi$ can be estimated using the NOS and NOP obtained from the ART. The probability of $\phi$ being less than or equal to the rejection level $\alpha$ is the probability that $\phi$ obtained from a beta distribution, with parameters NOS +1 and NOP $-\mathrm{NOS}+1$, is less than or equal to $\alpha$. The $\alpha$ can be set at either the .01 or .05 level to evaluate the probability of $\phi$ being less than or equal to the chosen levels. For example, if the ART with NOP $=1,000$ results in NOS $=52$, the probabilities for $\phi$ being less than .01 and .05 are near 0 and 0.44 , respectively. If these probabilities are quite large (e.g., larger than .95), then we can confidently reject the null hypothesis at the $\alpha$ level. The probabilities in this example are too small to let us confidently reject the null hypothesis. This additional refinement by Noreen can be used when the number of possible permutations of the data set is greater than the NOP.

It should be noted that the specific hypothesis tested by the ART is associated with the statistic selected. In general, a hypothesis tested by a parametric method using a conventional statistic such as $t$ or $F$ can also be tested by the ART using the same statistic. For instance, a $t$ test for mean difference for two independent groups can be replaced by the ART using $t$ as the statistic. In practice, however, the equivalent test statistics, which are relatively simpler to calculate, are usually used to obtain the same results. For example, the ART using mean difference as the statistic will obtain exactly the same $p$ value as the ART using $t$ as the statistic because the number of significant cases is the same using the two statistics based on the same pseudosamples (Edgington, 1987, p. 45; Manly, 1991, p. 16). Detailed discussion about selecting an equivalent test statistic for the ART is provided by Edgington and Manly.

ART procedures are currently not available on commonly used computer statistical packages such as BMDP, SPSS, SAS, or MINITAB. This article presents an SAS program that tests the hypothesis for equality of two means using the ART. This program may serve as a template for other hypothesis testing with minor modification. Although FORTRAN programs for the ART are available elsewhere (e.g., Edgington, 1987; Noreen, 1989), the handy tools in SAS (SAS Institute, Inc., 1990) make the user-written code much simpler. For example, the SAS function PROBBETA directly returns a confidence level for the $p$ value obtained from the ART, while in FORTRAN programs this computation requires several subroutines (Noreen, 1989).

Program 1 (see Appendix) tests the null hypothesis of no mean difference between two groups with equal or unequal sample sizes. This program first reads the original data from an external file. An example of the data file is listed in Table 1, with $x$ representing group membership and $y$ representing the dependent variable. The statistic selected in this program is the mean difference of the two groups. The program then calculates the original statistic, creates 1,000 pseudosamples each with variable $y$ shuffled and $x$ unchanged, and computes the pseudostatistic for each pseudosample. The shuffling algorithm used in the program is the one with desired properties recommended by Castellan (1992). Finally, the program counts the NOS and prints out the original statistic, the estimated significance level, and the confidence levels for $\phi$ less than or equal to rejection levels at .05 and .01, respectively. The significance level printed out by this program is for a two-tailed test. For a one-tailed test, this value should be divided by 2 . The program ran on the CMS operating system on the IBM 3081, and $9.45 \mathrm{sec}$ of CPU time was used $(N=20)$. For $N=10$, 50,100 , and 200, the required CPU time is 6.23, 17.65, 28.46 , and $59.77 \mathrm{sec}$, respectively.

The data in Table 1 were provided by Edgington (1987, p. 95) to show the power superiority of the randomization test over Student's $t$ test for skewed data. The $t$ test results in $t(18)=-1.7802, p=.0919$. The WilcoxonMann-Whitney test using SAS Proc NPARIWAY (large sample approximation) results in $p=.0636$. The results from Program 1 showed $p=.033$, with a confidence level 
Table 1

Sample Data File for Program $1(N=20)$

\begin{tabular}{cc} 
Sample Data File for Program $1(\boldsymbol{N}=\mathbf{2 0})$ \\
\hline $\boldsymbol{x}$ & $y$ \\
1 & 0.33 \\
1 & 0.27 \\
1 & 0.44 \\
1 & 0.28 \\
1 & 0.45 \\
1 & 0.55 \\
1 & 0.44 \\
1 & 0.76 \\
1 & 0.59 \\
1 & 0.01 \\
2 & 0.28 \\
2 & 0.80 \\
2 & 3.72 \\
2 & 1.16 \\
2 & 1.00 \\
2 & 0.63 \\
2 & 1.14 \\
2 & 0.33 \\
2 & 0.26 \\
2 & 0.63 \\
\hline
\end{tabular}

of .99 at $\phi \leq .05$ and a confidence level near 0 at $\phi$ $\leq .01$, indicating that the null hypothesis can be confidently rejected at the .05 level but not at the .01 level.

No change of program code is required when testing a new data set if the total number of observations is less than 800 . For a data set with more than 800 observations, only the upper bound value of array Temp at the end of the second line in Dataset Shuffle needs to be changed. For example, for 900 total observations, "s800" in the program should be changed to " $\mathrm{s} 900$." This program can be used for all sample sizes. However, for small samples with the number of possible permutations less than 1,000 , this program is slightly less efficient than the exact permutation test because it generates more samples than the number of possible permutations. The number of possible permutations for two independent groups is

$$
\left(\begin{array}{c}
m+n \\
n
\end{array}\right)=\frac{(m+n) !}{m ! n !},
$$

where $m$ and $n$ are sample sizes for the two groups. For example, when $m=5$ and $n=4$, the number of possible permutations is $9 ! /(5 ! 4 !)=126$.

Program 1 can serve as a template to test other hypotheses with modification of the part of the code that calculates the statistic. An example is listed in Program 2 (see Appendix), which tests the significance of correlation between two variables. The natural statistic for the test is the Pearson product-moment correlation coefficient $r$. The input data file has two fields: $x$ for one variable, and $y$ for the other. The program creates pseudosamples with variable $y$ shuffled and $x$ remaining in the original order. As in Program 1, the $p$ value printed out by the program is for a two-tailed test. For a one-tailed test, this value should be divided by 2 .

Program 3 (see Appendix) tests the hypothesis about the equality of more than two means with equal or unequal group sizes. The appropriate statistic for the test is

$$
\sum_{i=1}^{k} T_{i}^{2} / n_{i},
$$

where $k$ is the number of groups, $T_{\mathrm{i}}$ is the sum of the scores in Group $\mathrm{i}$, and $n_{\mathrm{i}}$ is the number of subjects in Group i. This statistic leads to results equivalent to those obtained using $F$ as the statistic (Edgington, 1987). The data file is similar to that in Table 1: $x$ represents the group code, and $y$ represents the dependent variable.

Requests for the program code can be mailed to the first author.

\section{REFERENCES}

Bradbury, I. (1987). Analysis of variance versus randomization tests: A comparison. British Journal of Mathematical \& Statistical Psychology, 40, 177-187.

Bradley, J. V. (1968). Distribution-free statistical methods. New Jersey: Prentice-Hall.

BradLey, J. V. (1978). Robustness? British Joumal of Mathematical \& Statistical Psychology, 31, 144-152.

Castellan, N. J., JR. (1992). Shuffling arrays: Appearances may be deceiving. Behavior Research Methods, Instruments, \& Computers, 24, 72-77.

DiXON, W. J., MASSEY, F. J. (1969). Introduction to statistical analysis (3rd ed.). New York: McGraw-Hill.

EDGington, E. S. (1969). Approximate randomization tests. Journal of Psychology. 72, 143-149.

Edgington, E. S. (1987). Randomization tests. New York: Marcel Dekker.

FishER, R. A. (1935). The design of experiments. Edinburgh: Oliver \& Boyd

Games, P. A. (1984). Data transformation, power, and skew: A rebuttal to Levine and Dunlap. Psychological Bulletin, 95, 345-347.

HoefFDing, W. (1952). The large sample power of tests based on permutations of observations. Annals of Mathematical Statistics, 23, $169-192$

Kempthone, O., \& Doerfler, T. E. (1969). The behavior of some significance tests under experimental randomization. Biometrika, 56, $231-248$

MANLY, B. F. J. (1991). Randomization and Monte Carlo methods in biology. London: Chapman \& Hall.

MICCERI, T. (1989). The unicorn, the normal curve, and other improbable creatures. Psychological Bulletin, 105, 156-166.

NOREEN, E. W. (1989). Computer-intensive methods for testing hypotheses: An introduction. New York: Wiley.

SAS INSTITUTE, INC. (1990). SAS language (Ver. 6) [Computer program]. Gary, NC: Author.

Siegel, S. \& CaStellan, N. J., JR. (1988). Nomparametric statistics for the behavioral sciences (2nd ed.). New York: McGraw-Hill.

STILL, A. W., \& WhITE, A. P. (1981). The approximate randomization test as an alternative to the $F$ test in analysis of variance. British Journal of Mathematical \& Statistical Psychology, 34, 243-252.

TOOTHAKER, L. E. (1972). An empirical investigation of the permutation $t$ test. British Journal of Mathematical \& Statistical Psychology, 25, 83-94

\section{APPENDIX}

\section{Program 1}

An SAS Program for the ART Testing Equality of Two Means

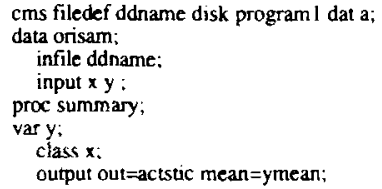




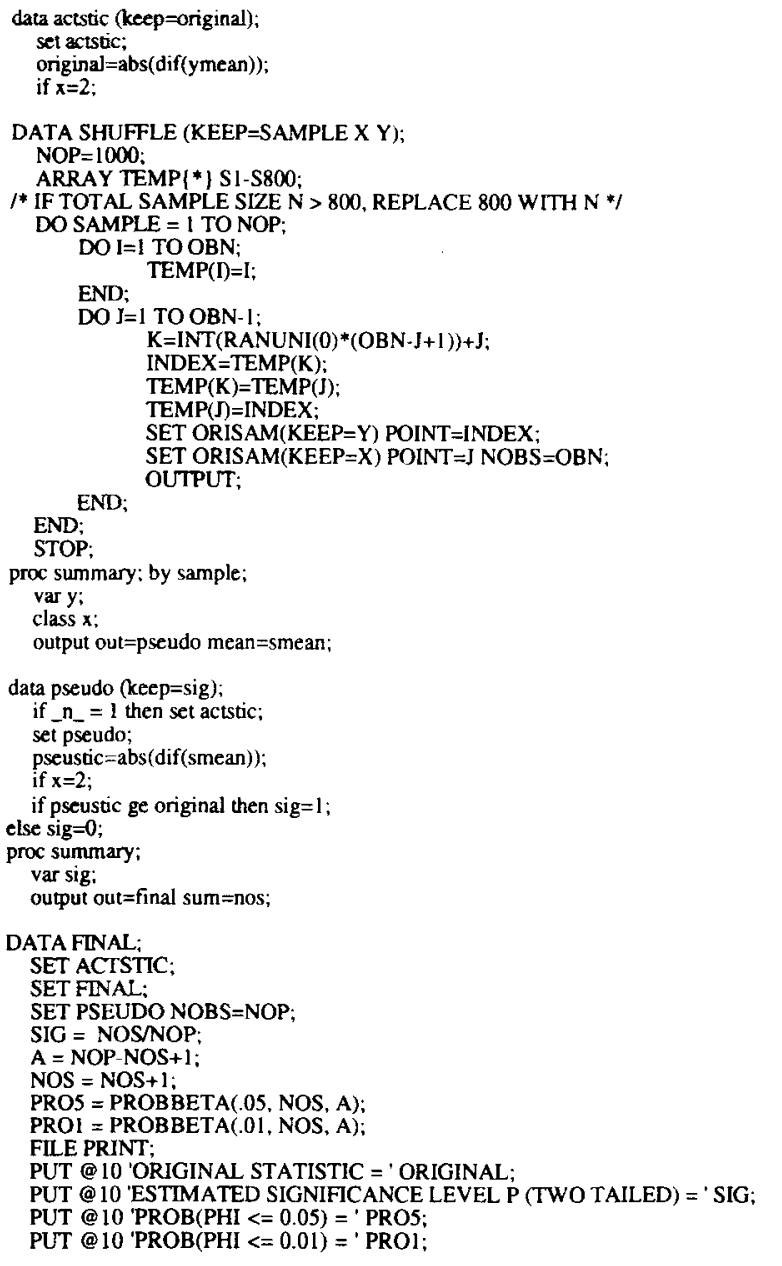

Note-The first line in this program is specific for CMS operating system to read external data file. The two blocks of listings in uppercase remain unchanged when testing other hypotheses. The first block is for shuffling the original data. The second block is for printing out the results.

\section{Program 2}

An SAS Program for the ART Testing Significance of Correlation

cms filedef ddname disk program2 dat a

data orisam;

infile ddname:

input $x y$;

proc corr outp=actstic noprint;

$$
\operatorname{var} x y \text {; }
$$

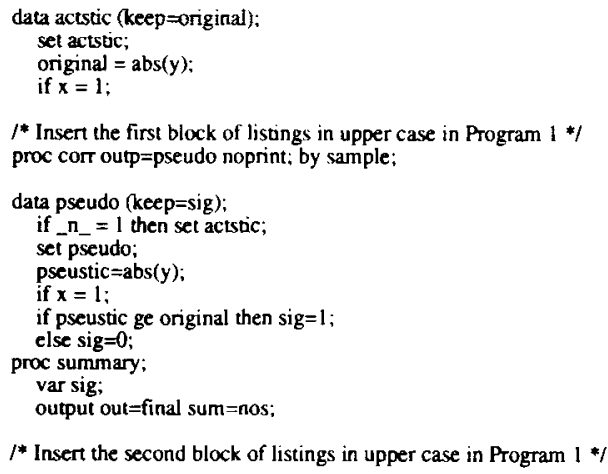

\section{Program 3}

An SAS Program for the ART Testing Equality of More Than Two Means

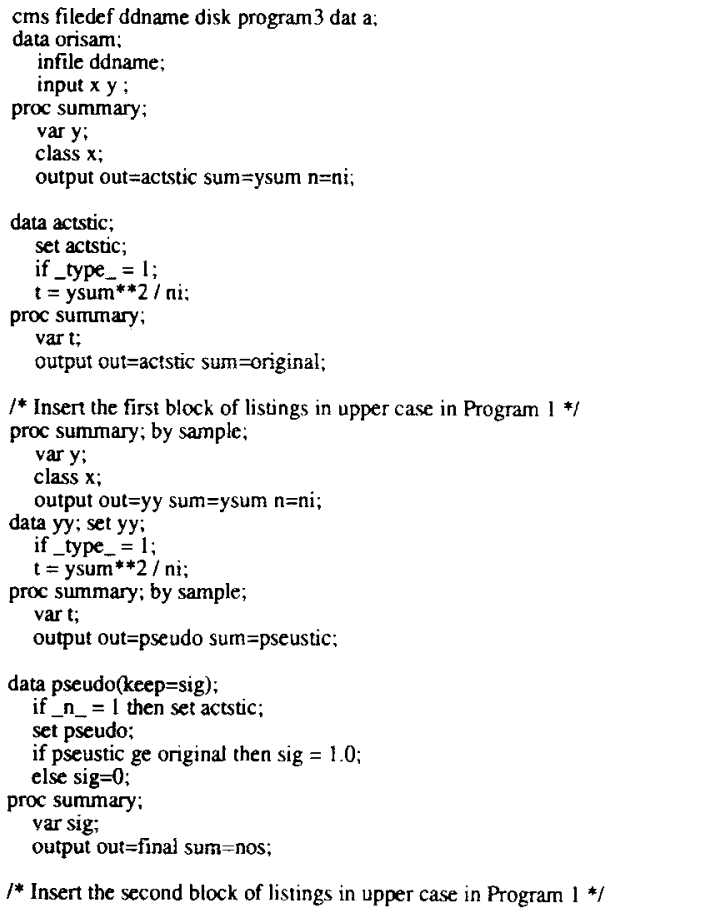

(Manuscript received June 28, 1991; revision accepted for publication December 10, 1992.) 